\title{
Approximate Fixed Point Sequences of an Evolution Family on a Metric Space
}

\author{
Si Fuan, ${ }^{1}$ Rizwan Ullah, ${ }^{2}$ Gul Rahmat $\left(\mathbb{D}^{2},{ }^{2}\right.$ Muhammad Numan, ${ }^{3}$ Saad Ihsan Butt $\mathbb{D}^{\mathbb{D}}{ }^{4}$ \\ and Xun $\mathbf{G e}^{5}$ \\ ${ }^{1}$ General Institute of Water Resources and Hydropower Planning and Design, Ministry of Water Resources Beijing, \\ Beijing 100120, China \\ ${ }^{2}$ Department of Mathematics, Islamia College Peshawar, Peshawar, KPK, Pakistan \\ ${ }^{3}$ Department of Mathematics, COMSATS University Islamabad, Attock, Pakistan \\ ${ }^{4}$ Department of Mathematics, COMSATS University Islamabad, Lahore, Pakistan \\ ${ }^{5}$ School of Electrical and Information Engineering, Zhengzhou University of Light Industry, Zhengzhou, \\ Henan Province 450002, China
}

Correspondence should be addressed to Saad Ihsan Butt; saadihsanbutt@cuilahore.edu.pk

Received 26 January 2020; Accepted 27 March 2020; Published 14 April 2020

Academic Editor: Ali Jaballah

Copyright (C) 2020 Si Fuan et al. This is an open access article distributed under the Creative Commons Attribution License, which permits unrestricted use, distribution, and reproduction in any medium, provided the original work is properly cited.

In this article, we study the approximate fixed point sequence of an evolution family. A family $E=\{U(x, y) ; x \geq y \geq 0\}$ of a bounded nonlinear operator acting on a metric space $(M, d)$ is said to be an evolution family if $U(x, x)=I$ and $U(x, y) U(y, z)=$ $U(x, z)$ for all $x \geq y \geq z \geq 0$. We prove that the common approximate fixed point sequence is equal to the intersection of the approximate fixed point sequence of two operators from the family. Furthermore, we apply the Ishikawa iteration process to construct an approximate fixed point sequence of an evolution family of nonlinear mapping.

\section{Introduction}

The study of an evolution equation is much more difficult but applicable for people working in this field of nonautonomous differential equation. The idea of an evolution equation arises from the solution of the nonautonomous Cauchy problem:

$$
\begin{aligned}
& \dot{y}(s)=A(s) y(s), \\
& y(0)=\xi .
\end{aligned}
$$

This article is about to explore the existence of approximate fixed point sequences for the subset of an evolution family of nonlinear operator acting on metric spaces. Fixed point theory is a muscular tool in various fields such as differential equations, economics, optimal control, game theory dynamical system [1-3]. In [4], Dehaish and Khamsi explore the existence of fixed points for the family of semigroups in metric spaces. In case of modular function spaces, various forms of fixed point conclusions for pointwise nonexpansive, asymptotic pointwise nonexpansive, pointwise contractions, and asymptotic pointwise contraction operators have been presented by Khamsi and Kozlowski $[5,6]$.

Since 1960, an extensive amount of work [7-9] has been devoted to fixed point theory, for the family of operator-like contractions and nonexpansive operators acting on Banach spaces as explored by Belluce and Kirk [10, 11], Bruck and Browder [12, 13], Lim [14], and DeMarr [15]. The asymptotic approach for the occurrence of common fixed points of semigroups of Lipschitzian operators has also been discussed, see the work done by Kirk and Xu [16]. Acting in Banach spaces, the existence of fixed points for asymptotic pointwise contraction operators and asymptotic pointwise nonexpansive operators is proved by Kirk and $\mathrm{Xu}$ in [16], and this result is extended to metric spaces by Hussain and Khamsi [17]. In [18], the author discussed, the intersection of fixed point sequence for nonexpansive semigroups of 
nonlinear operators $\{T(x): x \geq 0\}$, i.e., $T(0)=I$ and $T(x+$ $y)=T(x) T(y)$, for all $x \geq y \geq 0$ on a metric, and the Ishikawa iteration is given for approximation of the common fixed point sequence of the semigroups. In the current article, we will define the common fixed point sequence for evolution families of bounded linear mappings on metric space and then demonstrate the collection of common fixed point sequence for a subset of the evolution family in terms of two operators in the family. For applications of fixed point theory, we refer to the readers [19-23] and the references therein.

\section{Methodology}

The idea of evolution family of bounded linear operators arises from the solutions of the nonautonomous differential system. It is more general than the semigroup of bounded linear operators. In this paper, we generalize the results from the semigroup to the evolution family.

First, we define the family of evolution equation of bounded linear operators $E=\{U(x, y) ; x \geq y \geq 0\}$. Then, for the subset $L=\{U(t, 0) ; t \geq 0\}$ of this family of evolution equation, we prove that for any two positive real numbers $\alpha$ and $\beta$ such that $\alpha / \beta$ is irrational, the common approximate fixed point sequence is equal to the intersection of the approximate fixed point sequence of two operators from the family, that is,

$$
\operatorname{AFPS}(U(\alpha, 0)) \cap \operatorname{AFPS}(U(\beta, 0))=\operatorname{AFPS}(L) .
$$

Finally, applying the Ishikawa iteration process defined as $x_{n+1}=\alpha_{n} S\left(\beta_{n} T\left(x_{n}\right)+\left(1-\beta_{n}\right) x_{n}\right)+\left(1-\alpha_{n}\right) x_{n}$, we construct a sequence $\left\{x_{n}\right\}$ and we show that under certain restrictions, $\left\{x_{n}\right\}$ is an approximate fixed point sequence of both operators $T$ and $S$.

\section{Main Results}

Definition 1 (see [18]). If $\Omega$ is a nonempty subset of $(\Re, d)$, an operator $T: \Omega \mathrm{x} \longrightarrow \Omega$ is called nonexpansive if for any $s, t \in \Omega$, the following inequality

$$
d(T(s), T(t)) \leq d(s, t),
$$

holds. If $T(s)=s$ for any $s \in \Omega$, then the point $s$ is said to be the fixed point of the operator $T$, where $F(T)$ is used to represent the collection of all fixed points of operator $T$. A sequence $\left\{x_{n}\right\}$ in $\Omega$ is called an approximate fixed point sequence of the operator $T$ if $\lim _{n \longrightarrow \infty} d\left(T\left(x_{n}\right), x_{n}\right)=0$. The collection of approximate fixed point sequences of operator $T$ is given by $\operatorname{AFPS}(T)$.

Definition 2 A family $\{T(t): t=0\}$ of mappings is called a one-parameter continuous semigroup of nonexpansive mappings on a nonempty closed convex subset $C$ of a Banach space $X$ if the following conditions hold:

(i) For each $t \in \mathscr{R}^{+}, T(t)$ is a nonexpansive mapping on $C$;

(ii) $T(s+t)=T(t) \circ T(s)$, for all $t, s \in \mathscr{R}^{+}$; (iii) For each $x \in X$, the mapping $T(\cdot) x$ from $\mathscr{R}^{+}$into $C$ is continuous.

In the case of Banach spaces, a nonexpansive operator defined on the subset, which is nonempty, convex, closed, and bounded, has an approximate fixed point sequence rather than a fixed point. Now, we discuss this concept in the family of evolution equations.

Definition 3. A family of bounded linear operator $E=\{U(x, y) ; x \geq y \geq 0\}$ on a Banach space $X$ is called an evolution family if

(i) $U(x, x)=I$ for all $x \geq 0$.

(ii) $U(x, y) U(y, z)=U(x, z)$ such that $x \geq y \geq z \geq 0$. Such a family is called nonexpansive, continuous, strongly continuous, and periodic if

(iii) $\|U(x, y) s-U(x, y) t\| \leq\|s-t\| \forall x \geq y \geq 0, \quad$ and $s, t \in \Omega$.

(iv) $\lim _{x-y \longrightarrow 0} U(x, y) s=s \forall s \in \Omega$.

(v) For any nonempty and bounded subset $k \subset \Omega$, we have $\lim _{(x, y) \longrightarrow(0,0)} \sup _{s \in K} U(x, y) s=s$.

(iv) There exists $\quad p \geq 0$ such that $U(x+p, y+p)=U(x, y)$ for all $x \geq y \geq 0$, exists, respectively.

In the current article, we will assume the subset of an evolution family of bounded linear mapping on a Banach space $X$, define by $L=\{U(x, 0) ; x \geq 0\}$ which is $\alpha$ and $\beta$ periodic. Now, we define the collection of an approximate fixed point sequence of the family $E$ and $L$ as

$$
\begin{aligned}
& \operatorname{AFPS}(E)=\bigcap_{x \geq y \geq 0} \operatorname{AFPS}(U(x, y)), \\
& \operatorname{AFPS}(L)=\bigcap_{x \geq 0} \operatorname{AFPS}(U(x, 0)) .
\end{aligned}
$$

Proposition 1 (see [24]). If $W \subset R$ is a nonempty and additive subgroup, then either $\bar{W}=R$ or there exists a number $k$, which is nonnegative such that $W=k \cdot Z=\{k n, n \in Z\}$. If there exists two real numbers $\alpha$ and $\beta$ such that their fraction $\alpha / \beta$ is irrational, then the collection

$$
W(\alpha, \beta)=\{\alpha n+\beta m ; n, m \in Z\},
$$

is dense in the set $R$. Moreover, if $D=W(\alpha, \beta) \cap[0,+\infty)$ then $\bar{D}=[0,+\infty)(D$ is dense in $[0,+\infty))$.

Lemma 1. If $\Omega \subset(\Re, d)$ such that $\Omega$ is nonempty, and if $T: \Omega \longrightarrow \Omega$ is a nonexpansive operator, then for any $m \geq 2$, we have $\operatorname{AFPS}(T) \subset \operatorname{AFPS}\left(T^{m}\right)$.

Proof. Without the loss of generality, we consider that $\operatorname{AFPS}(T) \neq \varnothing$. Let $\quad\left\{x_{n}\right\} \in \operatorname{AFPS}(T) . \quad$ Then, $\lim _{n \longrightarrow \infty} d\left(T\left(x_{n}\right), x_{n}\right)=0$. Let fix $m \geq 2$, then

$$
d\left(T^{m}\left(x_{n}\right), x_{n}\right) \leq \sum_{k=1}^{m} d\left(T^{k}\left(x_{n}\right), T^{k-1}\left(x_{n}\right)\right) \leq m d\left(T\left(x_{n}\right), x_{n}\right),
$$


for any $n$ greater or equal to 1 . As $m$ is fixed, therefore, $\left\{x_{n}\right\} \in \operatorname{AFPS}(T)$, and we get

$$
\lim _{n \longrightarrow \infty} d\left(T^{m}\left(x_{n}\right), x_{n}\right)=0,
$$

hence $\left\{x_{n}\right\} \in \operatorname{AFPS}(T)$.

Lemma 2. If $\Omega \subset(\Re, d)$ such that $\Omega$ is nonempty. Let $L=$ $\{U(t, 0) t \geq 0\}$ be a subset of an evolution family acting on $\Omega$. If $\alpha$ and $\beta$ are two positive real numbers, which are also the periods of this family. Then,

$$
\operatorname{AFPS}(U(\alpha, 0)) \cap \operatorname{AFPS}(U(\beta, 0)) \subset \cdot \bigcap_{t \in W} \operatorname{AFPS}(U(t, 0)) .
$$

Proof. Without the loss of generality, we consider $\operatorname{AFPS}(U(\alpha, 0)) \cap \operatorname{AFPS}(U(\beta, 0)) \neq \phi$. Let $\left\{x_{n}\right\} \in \operatorname{AFPS}(U$ $(\alpha, 0)) \cap \operatorname{AFPS}(U(\beta, 0))$.

Recall that $W(\alpha, \beta)=\{\alpha n+\beta m ; n, m \in Z\}$ if $t \in W$, and by definition of $W, t=m \alpha+n \beta$ where $m, n \geq 0$. Then,

$$
\begin{aligned}
d\left(U(t, 0) x_{n}, x_{n}\right) & =d\left(U(m \alpha+n \beta, 0) x_{n}, x_{n}\right) \\
& =d\left(U^{m}(\alpha, 0) U^{n}(\beta, 0) x_{n}, x_{n}\right) .
\end{aligned}
$$

It means that

$$
\begin{aligned}
d\left(U(t, 0) x_{n}, x_{n}\right) \leq & d\left(U^{m}(\alpha, 0) U^{n}(\beta, 0) x_{n}, U^{m}(\alpha, 0) x_{n}\right) \\
& +d\left(U^{m}(\alpha, 0) x_{n}, x_{n}\right) \\
\leq & d\left(U^{n}(\beta, 0) x_{n}, x_{n}\right)+d\left(U^{m}(\alpha, 0) x_{n}, x_{n}\right),
\end{aligned}
$$

for any $n$ greater or equal to 1 . According to Lemma 1 , we get $\lim _{n \rightarrow \infty} d\left(U(t, 0) x_{n}, x_{n}\right)=0$, which implies $\left\{x_{n}\right\} \in$ $\operatorname{AFPS}(U(t, 0))$. Next, consider $\alpha n+\beta m$ such that $n$ or $m$ is negative. Without the loss of generality, consider $\alpha n-\beta m ; m, n \geq 0$. Then, we have

$$
\begin{aligned}
& d\left(U(t, 0) x_{n}, x_{n}\right)=d\left(U(m \alpha-n \beta, 0) x_{n}, x_{n}\right) \\
& \leq d\left(U(m \alpha-n \beta, 0) x_{n}, U(m \alpha, 0) x_{n}\right)+d\left(U(m \alpha, 0) x_{n}, x_{n}\right) .
\end{aligned}
$$

Since $U(m \alpha, 0)=U(m \alpha-n \beta, 0) \circ U(n \beta, 0)$ and $U(m \alpha-$ $n \beta, 0)$ are nonexpansive, we get

$$
\begin{aligned}
d\left(U(t, 0) x_{n}, x_{n}\right) & \leq d\left(x_{n}, U(n \beta, 0) x_{n}\right)+d\left(U(m \alpha, 0) x_{n}, x_{n}\right) \\
& =d\left(x_{n}, U^{n}(\beta, 0) x_{n}\right)+d\left(U^{m}(\alpha, 0) x_{n}, x_{n}\right),
\end{aligned}
$$

for any $n$ greater or equal to 1 . Again by Lemma 1 , we have $\lim _{n \rightarrow \infty} d\left(U(t, 0) x_{n}, x_{n}\right)=0$ which implies $\left\{x_{n}\right\} \in$ AFPS $(U(t, 0))$. Hence,

$$
\left\{x_{n}\right\} \in \bigcap_{t \in W} \operatorname{AFPS}(U(t, 0)) .
$$

Theorem 1. $\Omega$ is a subset of the metric space $(\Re, d)$ which is nonempty and bounded. Let $L=\{U(t, 0) t \geq 0\}$ be a subset of an evolution family of nonexpansive operators from $\Omega$ into $\Omega$.
Consider that $L$ is strongly continuous. If $\alpha$ and $\beta$ are two numbers, which are positive and real and also periods of this family with the condition that $\alpha / \beta$ is irrational. Then,

$$
\operatorname{AFPS}(U(\alpha, 0)) \cap \operatorname{AFPS}(U(\beta, 0))=\operatorname{AFPS}(L) .
$$

Proof. Since

$$
\operatorname{AFPS}(L) \subset \operatorname{AFPS}(U(\alpha, 0)) \cap \operatorname{AFPS}(U(\beta, 0)),
$$

it is enough to prove that

$$
\operatorname{AFPS}(U(\alpha, 0)) \cap \operatorname{AFPS}(U(\beta, 0)) \subset \operatorname{AFPS}(L) .
$$

Without the loss of generality consider that $\operatorname{AFPS}(U(\alpha, 0)) \cap \operatorname{AFPS}(U(\beta, 0))$ is not empty. Let $\left\{x_{n}\right\} \in \operatorname{AFPS}(U(\alpha, 0)) \cap \operatorname{AFPS}(U(\beta, 0))$, and Lemma 2 implies that $\left\{x_{n}\right\} \in \cap_{t \in G} \operatorname{AFPS}(U(t, 0))$.

From Proposition $1, W_{+}(\alpha, \beta)=W(\alpha, \beta) \cap[0,+\infty)$ is dense in $[0,+\infty)$. Then, there exists $t_{m} \in W_{+}(\alpha, \beta)$ and $m \geq 1$ such that $\lim _{m \rightarrow \infty} t_{m}=t$. Then,

$$
\begin{aligned}
d\left(x_{n}, U(t, 0) x_{n}\right) & \leq d\left(x_{n}, U\left(t_{m}, 0\right) x_{n}\right)+d\left(U\left(t_{m}, 0\right) x_{n}, U(t, 0) x_{n}\right) \\
& \leq d\left(x_{n}, U\left(t_{m}, 0\right) x_{n}\right)+\sup d\left(U\left(t_{m}, 0\right) x, U(t, 0) x\right) .
\end{aligned}
$$

Let $\varepsilon>0$, and the family $L$ is continuous in strong sense, hence there exists $m_{0} \geq 1$ such that for any $m \geq m_{0}$, we have

$$
\sup d\left(U\left(t_{m}, 0\right) x, U(t, 0) x\right)<\varepsilon \text {. }
$$

Since $\left\{x_{n}\right\} \in \operatorname{AFPS}\left(U\left(t_{m_{0}}, 0\right)\right)$, from Lemma 2, there exists $n_{0} \geq 1$, such that

$$
d\left(x_{n}, U\left(t_{m_{0}}, 0\right) x_{n}\right) \leq \varepsilon,
$$

for any $n \geq n_{0}$. Hence,

$$
\begin{aligned}
& d\left(x_{n}, U(t, 0) x_{n}\right) \\
& \leq d\left(x_{n}, U\left(t_{m_{0}}, 0\right) x_{n}\right)+\sup d\left(U\left(t_{m_{0}}, 0\right) x, U(t, 0) x\right) \\
& \leq 2 \varepsilon
\end{aligned}
$$

for any $n \geq n_{0}$. Since $\epsilon$ was positive, hence we have

$$
\lim _{n \longrightarrow \infty} d\left(U(t, 0) x_{n}, x_{n}\right)=0,
$$

e.g., $\left\{x_{n}\right\} \in \operatorname{AFPS}(U(t, 0))$.

Corollary 1. If $\Omega$ is a subset of the metric space $(\Re, d)$ which is nonempty and bounded. Let $L=\{U(t, 0) t \geq 0\}$ be an evolution family of a nonexpansive operator from $\Omega$ into $\Omega$. Consider L is strongly continuous. Then,

$$
\begin{aligned}
& \operatorname{AFPS}(U(1,0)) \cap \operatorname{AFPS}(U(\sqrt{5}, 0)) \\
& =\operatorname{AFPS}(U(1,0)) \cap \operatorname{AFPS}(U(\pi, 0)) \\
& =\operatorname{AFPS}(L) .
\end{aligned}
$$

In the next section, we discuss a technique for constructing an approximate fixed point sequence of two operators acting in a metric space. 


\section{Common Approximate Fixed Point Sequence of Two Nonexpansive Mappings}

Reich and Shafrir in [25] discuss hyperbolic metric space and intersection of an approximate fixed point sequence of two operators define on this space, see also [26]. Now, we will discuss the construction of such an approximate fixed point sequence.

Definition 4 (see [27]). If $(\mathfrak{R}, d)$ is a metric space, it is called convex, if for any $s, t$ in $\mathfrak{R}$ there exist a unique metric segment $[s, t] \in F$, and for a unique point $u$ of $[s, t]$ and $\omega \in[0,1]$, denoted by $(1-\omega) s+\omega t$, the following inequality holds:

$$
\begin{aligned}
& d(s, u)=\omega d(s, t), \\
& d(u, t)=(1-\omega) d(s, t),
\end{aligned}
$$

where $F$ is a family of segments of the points of $\mathfrak{R}$.

Definition 5 (see [25]). Let a metric space $(\mathfrak{R}, d)$ be convex, and it is called a hyperbolic metric space if for all $q, s, t$ in $\mathfrak{R}$ and $\omega \in[0,1]$, the inequality

$$
d(\omega q+(1-\omega) s, \omega q+(1-\omega) t) \leq(1-\omega) d(s, t),
$$

holds.

The subset $\Omega$ of a hyperbolic metric space $\Re$ is convex if for any $s, t$ in $\Omega$, the segment $[s, t]$ is also in $\Omega$.

Definition 6 (see [18]). If a metric space $(\mathfrak{R}, d)$ is hyperbolic, then for any $k \in \mathfrak{R}$ and every positive real number $r$ and $\varepsilon>0$, if the inequality

$$
\begin{aligned}
& \delta(r, \varepsilon)=\inf \left\{1-\frac{1}{r} d\left(\frac{1}{2} s+\frac{1}{2} t, k\right),\right. \\
& d(s, k) \leq r, d(t, k) \leq r, d(s, t) \geq r \varepsilon\}>0,
\end{aligned}
$$

holds then it is uniformly convex.

The idea of uniform convexity was initially studied in Banach spaces [28]. This idea was generalized to metric spaces in [29]. One can also see $[25,26,30]$.

Lemma 3. If $(\mathfrak{R}, d)$ is a hyperbolic space which is uniformly convex, then for $r>0,0 \leq \mu \leq 1$, and positive numbers $\varepsilon>0$, we have

$$
d(a, \mu s+(1-\mu) t) \leq r(1-\delta(r, 2 \min \{\mu, 1-\mu\} \varepsilon)),
$$

for all $a, s, t \in \mathfrak{R}$, such that $d(z, s) \leq r, d(z, t) \leq r$, and $d(s, t) \geq r \varepsilon$.

Lemma 4. If a hyperbolic metric space $(\mathfrak{R}, d)$ is uniformly convex. Consider we have $v \in[0,+\infty)$ such that

$$
\begin{array}{r}
\lim _{n \longrightarrow \infty} \sup d\left(x_{n}, a\right) \leq v, \\
\lim _{n \longrightarrow \infty} \sup d\left(y_{n}, a\right) \leq v, \\
\lim _{n \longrightarrow \infty} d\left(a, \alpha_{n} x_{n}+\left(1-\alpha_{n}\right) y_{n}\right)=v,
\end{array}
$$

where $\alpha_{n} \in[\alpha, \beta]$ such that $0<\alpha \leq \beta<1$. Then, $\lim _{n \rightarrow \infty} d\left(x_{n}, y_{n}\right)=0$.

Let $\Omega$ be a subset of a hyperbolic metric space $(\Re, d)$ which is nonempty and convex. Let $S, T: \Omega \longrightarrow \Omega$ be two operators. Fix $x_{1} \in C$. The strong convergence of Ishikawa iterates $\left\{x_{n}\right\}$ is defined by

$$
x_{n+1}=\alpha_{n} S\left(\beta_{n} T\left(x_{n}\right)+\left(1-\beta_{n}\right) x_{n}\right)+\left(1-\alpha_{n}\right) x_{n},
$$

as given by Das and Debata, where $\alpha_{n}, \beta_{n} \in[0,1]$. Under certain restrictions, we will show that $\left\{x_{n}\right\}$ is an approximate fixed point sequence of both operators $T$ and $S$. Consider $S$ and $T$ as nonexpansive, and $p \in \Omega$ is their common fixed point. Then,

$$
\begin{aligned}
& d\left(x_{n+1}, p\right)=d\left(\alpha_{n} S\left(y_{n}\right)+\left(1-\alpha_{n}\right) x_{n}, p\right) \\
& \leq \alpha_{n} d\left(S\left(y_{n}\right), p\right)+\left(1-\alpha_{n}\right) d\left(x_{n}, p\right) \\
& \leq \alpha_{n} d\left(y_{n}, p\right)+\left(1-\alpha_{n}\right) d\left(x_{n}, p\right) \\
& =\alpha_{n} d\left(\beta_{n} T\left(x_{n}+\left(1-\beta_{n}\right) x_{n}\right), p\right)+\left(1-\alpha_{n}\right) d\left(x_{n}, p\right) \\
& \leq \alpha_{n}\left[\beta_{n} d\left(T\left(x_{n}, p\right)+\left(1-\beta_{n}\right) d\left(x_{n}\right), p\right)\right]+\left(1-\alpha_{n}\right) d\left(x_{n}, p\right) \\
& \leq d\left(x_{n}, p\right),
\end{aligned}
$$

where $y_{n}=\beta_{n} T\left(x_{n}+\left(1-\beta_{n}\right) x_{n}\right)$ which shows that the sequence $\left\{\left(x_{n}, p\right)\right\}$ is decreasing, hence $\lim _{n \rightarrow \infty} d\left(x_{n}, p\right)$ exists. Base on the above inequalities, we have

$$
\begin{aligned}
\lim _{n \longrightarrow \infty} d\left(x_{n}, p\right) & =\lim _{n \longrightarrow \infty} d\left(\alpha_{n} S\left(y_{n}\right)+\left(1-\alpha_{n}\right) x_{n}, p\right) \\
& =\lim _{n \longrightarrow \infty}\left[\alpha_{n} d\left(S\left(y_{n}\right), p\right)+\left(1-\alpha_{n}\right) d\left(x_{n}, p\right)\right]+\lim _{n \longrightarrow \infty}\left[\alpha_{n} d\left(y_{n}, p\right)+\left(1-\alpha_{n}\right) d\left(x_{n}, p\right)\right] \\
& =\lim _{n \longrightarrow \infty}\left[\alpha_{n} d\left(\beta_{n} T\left(x_{n}+\left(1-\beta_{n}\right) x_{n}\right), p\right)+\left(1-\alpha_{n}\right) d\left(x_{n}, p\right)\right] \\
& =\lim _{n \rightarrow \infty}\left[\alpha_{n}\left[\beta_{n} d\left(T\left(x_{n}, p\right)+\left(1-\beta_{n}\right) d\left(x_{n}\right), p\right)\right]+\left(1-\alpha_{n}\right) d\left(x_{n}, p\right) .\right.
\end{aligned}
$$

Theorem 2. $\Omega \subset \Re$ where it is a hyperbolic metric space, which is also completely uniformly convex, and $\Omega$ is nonempty, convex, and closed. Let $T, S: \Omega \longrightarrow \Omega$ be two mappings, such that Fix $(T) \cap$ Fix $(S) \neq \varnothing$ Fix $x_{1} \in \Omega$ and generate $\left\{x_{n}\right\}$ in $\Omega$ by (1) such that $\alpha_{n}$ and $\beta_{n}$ belong to $[\alpha, \beta]$ with $0<\alpha \leq \beta<1$, and then we have $\lim _{n \longrightarrow \infty} d\left(x_{n}, S\left(x_{n}\right)\right)=0$ and $\lim _{n \longrightarrow \infty} d\left(x_{n}, T\left(x_{n}\right)\right)=0$. 
Proof. Let $p \in \operatorname{Fix}(T) \cap \operatorname{Fix}(S)$. Then, $\left\{d\left(x_{n}, p\right)\right\}$ is a decreasing sequence. Fix $k=\lim _{n \rightarrow \infty} d\left(x_{n}, p\right)$. Now, in case for $k=0$, then all results are obvious. Therefore, we assume $k>0$. Then,

$$
\begin{aligned}
& d\left(x_{n+1}, p\right) \leq \alpha_{n} d\left(S\left(y_{n}\right), p\right)+\left(1-\alpha_{n}\right) d\left(x_{n}, p\right), \\
& d\left(S\left(y_{n}\right), p\right) \leq d\left(y_{n}, p\right), \\
& \leq \beta_{n} d\left(T\left(x_{n}\right), p\right)+\left(1-\beta_{n}\right) d\left(x_{n}, p\right) \leq d\left(x_{n}, p\right),
\end{aligned}
$$

where $n \geq 1$. By the above two inequalities, we have

$$
\begin{aligned}
& d\left(x_{n+1}, p\right)=d\left(\alpha_{n} S\left(y_{n}\right)+\left(1-\alpha_{n}\right) x_{n}, p\right) \\
& \leq \alpha_{n} d\left(S\left(y_{n}\right), p\right)+\left(1-\alpha_{n}\right) d\left(x_{n}, p\right) \leq d\left(x_{n}, p\right),
\end{aligned}
$$

which means $\lim _{n \longrightarrow \infty} d\left(S\left(x_{n}\right), p\right)=k$. Therefore, we get

$$
\begin{aligned}
& \lim _{n \longrightarrow \infty} d\left(S\left(y_{n}\right), p\right)=\lim _{n \longrightarrow \infty} d\left(S\left(x_{n}\right), p\right) \\
& =\lim _{n \longrightarrow \infty} d\left(\alpha_{n} S\left(y_{n}\right)\right)+\left(\left(1-\alpha_{n}\right) x_{n}, p\right)=k .
\end{aligned}
$$

By Lemma 4 , we conclude that $\lim _{n \longrightarrow \infty} d\left(S\left(y_{n}\right), x_{n}\right)=0$. Next, from (2) and (3), we get

$$
d\left(x_{n+1}, p\right) \leq \alpha_{n} d\left(y_{n}, p\right)+\left(1-\alpha_{n}\right) d\left(x_{n}, p\right) \leq d\left(x_{n}, p\right),
$$

which implies that $\lim \left[\alpha_{n} d\left(y_{n}, p\right)+\left(1-\alpha_{n}\right) d\left(x_{n}, p\right)\right]=k$. Since $\liminf \alpha_{n} \geq \alpha>0$, we have $\lim d\left(y_{n}, p\right)=k$. Since $\beta_{n} \geq \alpha>n \overrightarrow{0}$, we get $\lim _{n \longrightarrow \infty} d\left(T\left(x_{n}\right), \vec{p}\right) \stackrel{\infty}{=} k$ similarly. We have

$$
\begin{aligned}
& \lim _{n \longrightarrow \infty} d\left(x_{n}, p\right)=\lim _{n \longrightarrow \infty} d\left(T\left(x_{n}\right), p\right) \\
& =\lim _{n \longrightarrow \infty} d\left(\beta_{n} T\left(x_{n}\right)+\left(1-\beta_{n} x_{n}\right), p\right)=k .
\end{aligned}
$$
since

Using Lemma 4, we get $\lim _{n \rightarrow \infty} d\left(T\left(x_{n}\right), x_{n}\right)=0$. Finally,

$$
\begin{aligned}
d\left(x_{n}, S\left(x_{n}\right)\right) & \leq d\left(x_{n}, S\left(y_{n}\right)\right)+d\left(S\left(y_{n}\right), S\left(x_{n}\right)\right) \\
& \leq d\left(x_{n}, S\left(y_{n}\right)\right)+d\left(y_{n}, x_{n}\right) \\
& =d\left(x_{n}, S\left(y_{n}\right)\right)+\beta_{n} d\left(T\left(x_{n}\right), x_{n}\right) \\
& \leq d\left(x_{n}, S\left(y_{n}\right)\right)+d\left(T\left(x_{n}\right), x_{n}\right),
\end{aligned}
$$

we conclude that $\lim _{n \longrightarrow \infty} d\left(x_{n}, S\left(x_{n}\right)\right)=0$.

Remark 1. It is difficult to find a common fixed point of operators $S$ and $T$. If $S \circ T=T \circ S$, it means that the operators $S$ and $T$ commute, i.e., by Theorem 2 and $\Omega$ is which is bounded, the operators $S$ and $T$ have a common fixed point. Indeed, fix $x_{0} \in \Omega$ and define

$$
T_{n}(x)=\frac{1}{n} x_{0}+\left(1-\frac{1}{n}\right) T(x),
$$

for $x \in \Omega$ and $n \geq 1$. Consider

$$
\begin{aligned}
d\left(T_{n}(x), T_{n}(y)\right) & =d\left(\frac{1}{n} x_{0}+\left(1-\frac{1}{n}\right) T(x), \frac{1}{n} x_{0}+\left(1-\frac{1}{n}\right) T(y)\right) \\
& \leq\left(1-\frac{1}{n}\right) d\left(T_{n}(x), T_{n}(y)\right) \\
& \leq\left(1-\frac{1}{n}\right) d(x, y),
\end{aligned}
$$

where $x$ and $y$ belong to $\Omega$. Which show that, $T_{n}$ is a contraction. Then, $u_{n}$ in $\Omega$ is the unique fixed point of $T_{n}$ by the Banach contraction principle. As $\Omega$ is bounded and

$$
\begin{aligned}
d\left(u_{n}, T\left(u_{n}\right)\right) & =d\left(\frac{1}{n} x_{0}+\left(1-\frac{1}{n}\right) T\left(u_{n}\right), T\left(u_{n}\right)\right) \\
& \leq \frac{1}{n} d\left(x_{0}, T\left(u_{0}\right)\right),
\end{aligned}
$$

we have $d\left(u_{n}, T\left(u_{n}\right)\right) \longrightarrow 0$. Introduce the mapping

$$
\eta(x)=\lim _{n \longrightarrow \infty} \sup d\left(u_{n}, x\right) .
$$

As $\Omega$ is uniformly convex, $\sigma \in \Omega$ is a minimum point of $\eta$ which is also unique:

$$
\eta(\sigma)=\inf \{\eta(x) ; x \in \Omega\},
$$

and $\eta(\sigma)<\eta(x)$. For any $x \neq \sigma$, since the operator $T$ has an approximate fixed point sequence $\left\{u_{n}\right\}$, we get

$$
\begin{aligned}
\lim _{n \longrightarrow \infty} \sup d\left(u_{n}, T(\sigma)\right) & =\lim _{n \longrightarrow \infty} \sup d\left(T\left(u_{n}\right), T(\sigma)\right) \\
& \leq \lim _{n \longrightarrow \infty} \sup d\left(u_{n}, \sigma\right) .
\end{aligned}
$$

Therefore, $\eta(T(\sigma)) \leq \eta(\sigma)$ this gives $\sigma=T(\sigma)$. As the collection $\Omega$ is strictly convex, $\operatorname{Fix}(T)$ is a subset of $\Omega$ which is nonempty and convex. Also, the operators $S$ and $T$ commute, hence $S(\operatorname{Fix}(T)) \subset \operatorname{Fix}(T)$. From above, it is clear that $S$ has a fixed point in Fix $(T)$, which means that operators $S$ and $T$ have a fixed point in common.

Remark 2. If an evolution family is periodic of every period $p \geq 0$, then $L=\{U(x, 0): x \geq 0\}$ becomes a semigroup. Hence, paper [18] become a special case of our work.

\section{Conclusions}

The study of an evolution equation is much more difficult but applicable for people working in this field of nonautonomous differential equation. The idea of evolution family of bounded linear operators arises from the solutions of nonautonomous differential. In this paper, we introduce a sequence of an evolution family, which is more general than the semigroup of bounded linear operators. We proved that the common approximate fixed point sequence is equal to the intersection of an approximate fixed point sequence of two operators from the family. Furthermore, we apply the Ishikawa iteration process to construct an approximate fixed point sequence of an evolution family of nonlinear mapping. Interesting readers can use other iterations instead of Ishikawa iteration and prove the results of this paper. 


\section{Data Availability}

All data required for this paper are included within this paper.

\section{Conflicts of Interest}

The authors declare that they have no conflicts of interest.

\section{Authors' Contributions}

All authors contributed equally to this paper.

\section{Acknowledgments}

This research was supported by the National Key R\&D Program of China (2016YFC0401801).

\section{References}

[1] M. Tesfaye, K. Koyas, and S. Gebregiorgis, "A coupled fixed point theorem for maps satisfying rational type contractive condition in dislocated quasi b-metric space," Open Journal of Mathematical Sciences, vol. 4, no. 1, pp. 27-33, 2020.

[2] A. Perveen, S. Kausar, and W. Nazeer, "Strong convergence theorems of common fixed points for a uniformly closed asymptotically family of countable quasi-Lipschitz mappings in Hilbert spaces," Open Journal of Mathematical Analysis, vol. 3, no. 1, pp. 1-6, 2019.

[3] S. F. A. Naqvi and M. S. Khan, "On the viscosity rule for common fixed points of two nonexpansive mappings in Hilbert spaces," Open Journal of Mathematical Sciences, vol. 1, no. 1, pp. 111-125, 2017.

[4] B. A. B. Dehaish and M. A. Khamsi, "Approximation common fixed points of semigroups in metric spaces," Fixed Point Theory and Applications, vol. 2015, no. 1, p. 51, 2015.

[5] M. A. Khamsi and W. M. Kozlowski, "On asymptotic pointwise contractions in modular function spaces," Nonlinear Analysis: Theory, Methods \& Applications, vol. 73, no. 9, pp. 2957-2967, 2010.

[6] M. A. Khamsi and W. M. Kozlowski, "On asymptotic pointwise nonexpansive mappings in modular function spaces," Journal of Mathematical Analysis and Applications, vol. 380, no. 2, pp. 697-708, 2011.

[7] E. Zinab, K. Koyas, and A. Girma, "A fixed point theorem for generalized weakly contractive mappings in b-metric spaces," Open Journal of Mathematical Sciences, vol. 4, no. 1, pp. 1-8, 2020.

[8] E. Bonyah, M. Ahmad, and I. Ahmad, "On the viscosity rule for common fixed points of two nonexpansive mappings in CAT(0) spaces," Open Journal of Mathematical Sciences, vol. 2, no. 1, pp. 39-55, 2018.

[9] Z. Bekri and S. Benaicha, "Existence of solution for a nonlinear fifth-order three-point boundary value problem," Open Journal of Mathematical Analysis, vol. 3, no. 2, pp. 125-136, 2019.

[10] L. Belluce and W. Kirk, "Fixed-point theorems for families of contraction mappings," Pacific Journal of Mathematics, vol. 18, no. 2, pp. 213-217, 1966.

[11] L. P. Belluce and W. A. Kirk, "Nonexpansive mappings and fixed-points in Banach spaces," Illinois Journal of Mathematics, vol. 11, no. 3, pp. 474-479, 1967.
[12] F. E. Browder, "Nonexpansive nonlinear operators in a Banach space," Proceedings of the National Academy of Sciences, vol. 54, no. 4, pp. 1041-1044, 1965.

[13] R. Bruck, "A common fixed point theorem for a commuting family of nonexpansive mappings," Pacific Journal of Mathematics, vol. 53, no. 1, pp. 59-71, 1974.

[14] T. C. Lim, "A fixed point theorem for families on nonexpansive mappings," Pacific Journal of Mathematics, vol. 53, no. 2, pp. 487-493, 1974.

[15] R. DeMarr, "Common fixed points for commuting contraction mappings," Pacific Journal of Mathematics, vol. 13, no. 4, pp. 1139-1141, 1963.

[16] W. A. Kirk and H.-K. Xu, "Asymptotic pointwise contractions," Nonlinear Analysis: Theory, Methods \& Applications, vol. 69, no. 12, pp. 4706-4712, 2008.

[17] N. Hussain and M. A. Khamsi, "On asymptotic pointwise contractions in metric spaces," Nonlinear Analysis: Theory, Methods \& Applications, vol. 71, no. 10, pp. 4423-4429, 2009.

[18] M. A. Khamsi, "Approximate fixed point sequences of nonlinear semigroups in metric spaces," Canadian Mathematical Bulletin, vol. 58, no. 2, pp. 297-305, 2015.

[19] Y. Zhao, N. Ali, A. U. Haq, and M. Abbas, "Further generalized hybrid mappings and common attractive points in CAT(0) spaces: a new iterative process," IEEE Access, vol. 7, pp. 115208-115213, 2019.

[20] D. Li, M. Tanveer, W. Nazeer, and X. Guo, "Boundaries of filled julia sets in generalized jungck mann orbit," IEEE Access, vol. 7, pp. 76859-76867, 2019.

[21] Y. C. Kwun, M. Tanveer, W. Nazeer, M. Abbas, and S. M. Kang, "Fractal generation in modified jungck-S orbit," IEEE Access, vol. 7, pp. 35060-35071, 2019.

[22] Y. C. Kwun, A. A. Shahid, W. Nazeer, S. I. Butt, M. Abbas, and S. M. Kang, "Tricorns and multicorns in noor orbit with $s$ convexity,” IEEE Access, vol. 7, pp. 95285-95292, 2019.

[23] Y. C. Kwun, M. Tanveer, W. Nazeer, K. Gdawiec, and S. M. Kang, "Mandelbrot and Julia sets via jungck-CR iteration with $s$-convexity," IEEE Access, vol. 7, pp. 12167-12176, 2019.

[24] I. Stewart and D. Tall, Algebraic Number Theory and Fermats Last Theorem, A K Peters Ltd., Natick, MA, USA, 3rd edition, 2002.

[25] S. Reich and I. Shafrir, "Nonexpansive iterations in hyperbolic spaces," Nonlinear Analysis: Theory, Methods \& Applications, vol. 15, no. 6, pp. 537-558, 1990.

[26] K. Goebel and S. Reich, "Uniform convexity, hyperbolic geometry, and nonexpansive mappings," in Monographs and Textbooks in Pure and Applied Mathematics, Vol. 83, Marcel Dekker, New York, NY, USA, 1984.

[27] K. Menger, "Untersuchungen über allgemeine metrik," Mathematische Annalen, vol. 100, no. 1, pp. 75-163, 1928.

[28] J. A. Clarkson, "Uniformly convex spaces," Transactions of the American Mathematical Society, vol. 40, no. 3, p. 396, 1936.

[29] K. Goebel, T. Sekowski, and A. Stachura, "Uniform convexity of the hyperbolic metric and fixed points of holomorphic mappings in the Hilbert ball," Nonlinear Analysis: Theory, Methods \& Applications, vol. 4, no. 5, pp. 1011-1021, 1980.

[30] M. A. Khamsi and A. R. Khan, "Inequalities in metric spaces with applications," Nonlinear Analysis: Theory, Methods \& Applications, vol. 74, no. 12, pp. 4036-4045, 2011. 